\title{
Spiral artery remodeling in pregnancy
}

\begin{abstract}
Spiral arteries are the main arteries supplying the fetus during pregnancy. The secretion of Vascular Endothelial Growth Factor from the maternal decidua leads to the transformation of these vessels from high impedance vessels to capacitance vessels. Subsequently, there is trophoblastic proliferation and incorporation of fetal trophoblast cells into the maternal spiral arteriolar wall. This transformation is a fascinating series of events and forms the basis of intense Doppler ultrasound studies at the fetomaternal interphase.
\end{abstract}

Keywords: trophoblast, fetus, artery, pregnancy, ultrasound
Volume I Issue 3 - 2017

\section{Nidhi Sharma \\ Department of Obstetrics \& Gynaecology, Saveetha University, India}

Correspondence: Nidhi Sharma, Department of Obstetrics \& Gynaecology, Saveetha University, India, Email drbonuramkumar@yahoo.co.in
Abbreviations: JZ, junctional zone; VEGF, vascular endothelial growth factor; PLGF, placental growth factor

\section{Mini review}

In human placental bed, at the feto maternal interface, the extravillous trophoblastic cells invade not only the decidua but also the subendometrial or Junctional Zone (JZ) myometrium. ${ }^{1-3}$ The interstitial and endovascular migratory cells in the vessels wall were later confirmed to be trophoblastic in origin. ${ }^{4}$

Brosen et al. suggested that the "physiological change " of spiral arteries in the pregnant uteri was a result of the destructive action of invading trophoblast on vascular smooth muscles and elastic membranes. ${ }^{5}$ Later, a maternal contribution had to be considered since some changes in the maternal vessel wall precede the antidromic migration of trophoblast along the vessel lumen. Some researchers believe that the local intravasation of interstitial trophoblast is more likely. ${ }^{6}$

The four steps in which remodeling takes place is well documented (Figure 1) ${ }^{7,8}$ The first step is the decidua associated remodeling. Perivascular sheaths of swollen decidual cells (streeter's column) appear as early as postovulatory day $11 .^{3}$ These swollen perivascular cells may be derived from vascular smooth muscles. As early as 9 weeks the uterine decidual natural killer cells secrete Vascular Endothelial Growth Factor (VEGF), Placental growth Factor (PLGF) and angiopoietins. ${ }^{9,10}$

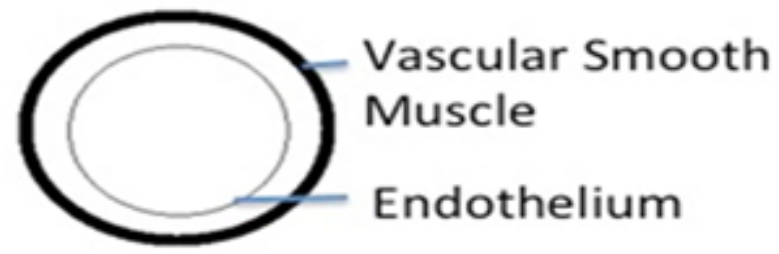

Figure IA Diagrammatic representation of unmodified spiral artery. Unmodified spiral artery showing endothelium and vascular smooth muscle.

This leads to vacuolation and disorganization of vascular intima and endothelial cells (Figure 1A \& 1B). In JZ myometrium, since the natural killer cells are absent, the presence of interstitially invading trophoblast may help the release of VEGF and angiopoietins ${ }^{9,10}$ This is evidenced by the fact that the interstitial trophoblast invades the JZ at 8 weeks.

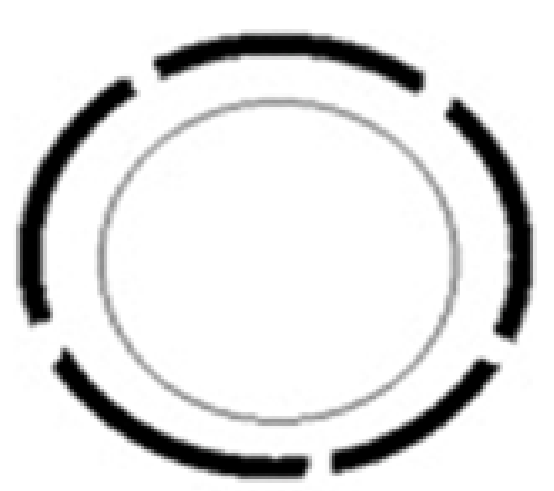

Figure IB Diagrammatic representation of spiral artery remodeling step I. Decidua associated remodeling with disorganization of vascular smooth muscles.

This is followed by the actual trophoblastic invasion and intraarterial migration (Figure 1C \& 1D). Invasion follows an interstitial and an endovascular course. The endovascular course happens in spiral arteries but never in veins. Interstitial trophoblast, but not endovascular, subsequently fuses to form multinuclear giant cells. ${ }^{11}$ Though the multinuclear giant cells appear more striking but it is the mononuclear cytotrophoblast that is most invasive and it occupies extensive area of uterine wall within a short time. An overwhelming number of basophilic mononuclear cells occupy the space between the smooth muscles of JZ myometrium. Quantitative study reveals that their distribution is at the center at 8 to 14 weeks and towards a biphasic distribution at 16-18 weeks. Thus they follow a ring like pattern towards the periphery of placental bed. ${ }^{12}$ It is thought that after their fusion to form giant cells they lose some potential of invasion. During endometrial decidualization a selective breakdown of extracellular matrix components occurs independent of trophoblastic action.

The interstitial invasion of decidua and JZ myometrium precedes the spiral artery invasion by several weeks. In early pregnancy mononuclear trophoblasts plug the outlets of spiral arterioles at the fetomaternal interface and thus create a low oxygen environment for the developing placenta and fetus. After 10 weeks the whole length of the spiral arteries in decidua may contain trophoblast reaching even up to the superficial vascular JZ Myometrium. Deep invasion of myometrial segments of the spiral arteries is not seen before 15 weeks. 


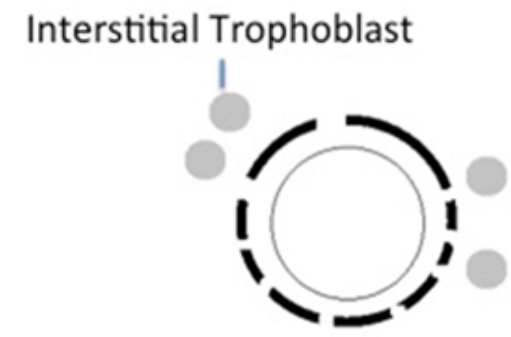

Figure IC Diagrammatic representation of spiral artery remodeling step 2. Interstitial Trophoblast migration enhances vascular smooth muscle disorganization.

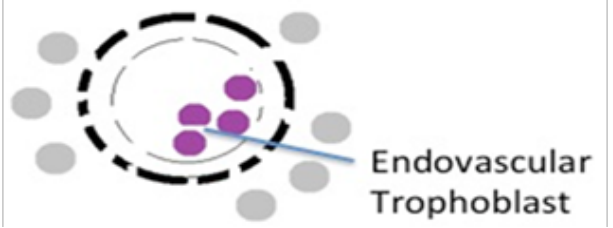

Figure ID Diagrammatic representation of spiral artery remodeling step 3. Endovascular Trophoblast temporarily replaces to endothelium.

The third step is trophoblast associated remodeling when the trophoblast cells are actually incorporated into the arterial wall (Figure 1E). This vascular incorporation is initiated by the penetration of the endothelium. Electron micrograph revealed that the trophoblast penetrates between the healthy endothelial cells and cross the underlying basement membrane. The smooth muscle penetration ultimately leads to its replacement by trophoblast embedded within a fibrinoid matrix, probably secreted by the trophoblast itself..$^{13}$



Figure IE Diagrammatic representation of spiral artery remodeling step 4. Intramural incorporation of endovascular trophoblast and deposition of fibrinoid, replacing the vascular smooth muscle.

The intraluminal trophoblasts now assume a spider like shape because of increasing accumulation of fibrinoid materials around the cell processes. As a rule, the intraluminal trophoblast remains mononuclear or at the most become binuclear. This is a contrast to the interstitial trophoblast.

The fourth step reendothelialization definitely occurs (Figure $1 \mathrm{~F})$. It is not clear whether the maternal vascular lining is repaired by endothelial remnants which were still present after the intramural invasion or whether a new endothelial covering is derived from circulating endothelial progenitor cells. ${ }^{14}$ The increase in placental oxygen tension has been correlated with trophoblastic migration (Figure 2)



Figure IF Diagrammatic representation of spiral artery remodeling step 4 . Reendothelialisation and intimal thickening.

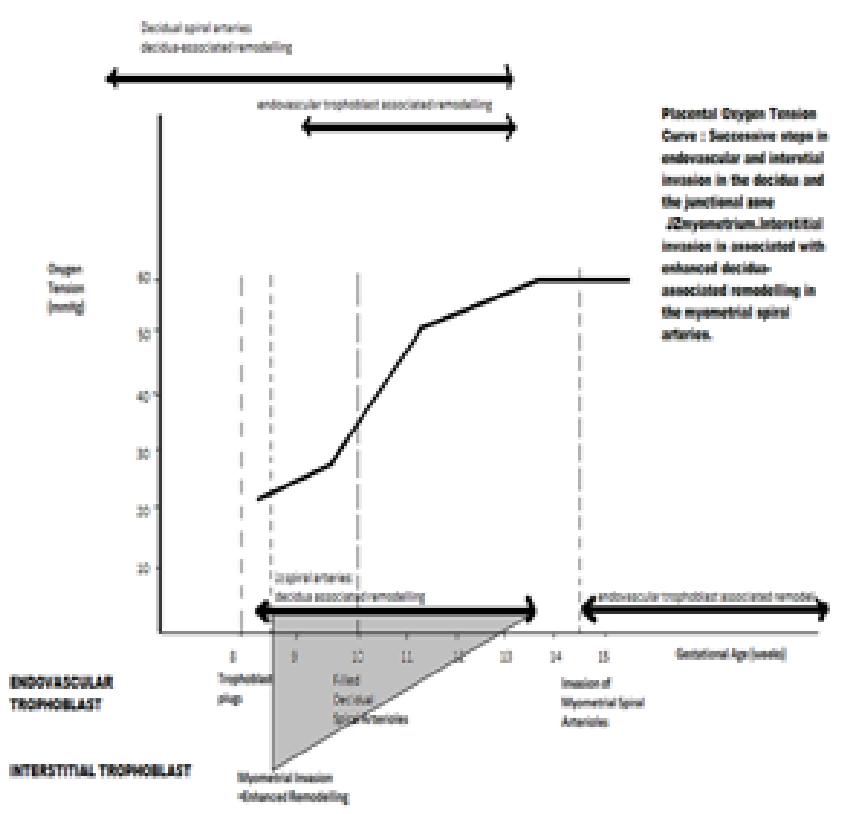

Figure 2 Placental oxygen tension curve: Successive steps in endovascular and interstitial invasion of decidua and the Junctional Zone endometrium. Interstitial invasion is associated with enhanced decidua associated remodeling in myometrial spiral arteries (shaded triangle).

\section{Acknowledgements}

Authors thank the staff of Department of obstetrics and Gynecology and Department of Radiology, Sri Ramachandra University and Medical College, Chennai, for the care given to the pregnant women. We also acknowledge the funds provided by Saveetha University, Chennai, Muthayammal scheme for the care of pregnant women.

\section{Conflict of interest}

The author declares no conflict of interest.

\section{References}

1. Hamilton WJ, Boyd JD. Development of the human placenta in the first three months of gestation. J Anat. 1960;94:297-328. 
2. Hamilton WJ, Boyd JD. Trophoblast in human placental arteries. Nature. 1966;212(5065):906-908.

3. Harris JWS, Ramsey EM. The morphology of human uteroplacental vasculature. Contributions to embryology. 1966;38:43-58.

4. Kaufmann P, Black S, Huppertz B. Endovascular trophoblast invasion: implications for the pathogeneses of intrauterine growth retardation and preeclampsia. Biol Reprod. 2003;69(1):1-7.

5. Brosens I, Robertson WB, Dixon HG. The physiological response of the vessels of placental bed to normal pregnancy. J Pathol Bacteriol. 1967;93(2):569-579.

6. Pijenborg R, Vercruysse L, Hanssens M. The uterine spiral arteries in human pregnancy: facts and controversies. Placenta. 1998;27(910):241-252.

7. Craven CM, Morgan T, Ward K. Decidual spiral artery remodeling begins before cellular interaction with cytotrophoblast. Placenta. 1998;19(4):241-252.

8. Xiao Feng Li, Stephen Charnock-Jones D, Eko Zhang, et al. Angiogenic growth factor messenger ribonucleic acids in uterine natural killer cells. J Clin Endocr Metab. 2001;86(4):1823-1834.
9. Brosens IA. Discussion. Eur J Obstet Gynaec Reprod Biol. 1975;5:4765

10. Pijenborg R, Bland JM, Robertson WB, et al. Uteroplacental arterial changes related to interstitial trophoblast migration in early human pregnancy. Placenta. 1983;4(4):397-414.

11. Bulmer JN, Lash GE. Human uterine natural killer cells: a reappraisal. Mol Immunol. 2005;42(4):511-521.

12. Schiessl B, Innes BA, Bulmer JN, et al. Localization of angiogenic growth factors and their receptors in the human placental bed throughout normal human pregnancy. Placenta. 2009;30(1):79-87.

13. De Wolf F, De Wolf-Peeters C, Brosens I, et al. The human placental bed: electron microscopic study of trophoblastic invasion of spiral arteries. Am J Obstet Gynecol. 1980;137(1):58-70.

14. Robb AO, Mills NL, Newby DE, et al. Endothelial progenitor cells in pregnancy. Reproduction. 2007;133(1):1-9. 